\title{
BMJ Open Prevalence and characteristics of psoriasis in Denmark: findings from the Danish skin cohort
}

\author{
Alexander Egeberg, Yuki M F Andersen, Jacob P Thyssen
}

To cite: Egeberg $A$, Andersen YMF, Thyssen JP. Prevalence and characteristics of psoriasis in Denmark: findings from the Danish skin cohort. BMJ Open 2019;9:e028116. doi:10.1136/ bmjopen-2018-028116

- Prepublication history and additional material for this paper are available online. To view these files, please visit the journal online (http://dx.doi. org/10.1136/bmjopen-2018028116).

Received 22 November 2018 Revised 1 February 2019 Accepted 26 February 2019

\begin{abstract}
Background Wide-ranging psoriasis prevalence estimates have been reported, possibly due to methodological differences.

Objectives To assess the prevalence of psoriasis in Denmark and to validate the use of questionnaire-based data to identify patients with psoriasis.

Methods We used data from the Danish Skin Cohort, a prospective cohort comprising general population adults, as well as patients with dermatologist-verified psoriasis and atopic dermatitis, respectively. The general population cohort was interviewed to assess the psoriasis prevalence in Denmark, and validation of the questions was performed.

Results From 3490 general population participants, 7.9\% $(n=275)$ were found to have self-reported psoriasis. Of these, 221 (prevalence 6.3\%) had their disease diagnosed by a physician (the dermatologist-diagnosed prevalence was $4.3 \%$ ), whereas 54 (prevalence $1.6 \%$ ) were not diagnosed by a physician. A total of $176(5 \%)$ had active psoriasis within the last 12 months. More than half of patients had at least one disease flare in the last 12 months, and $44.4 \%$ of patients with psoriasis had at least one family member with psoriasis, whereas this was only the case for $13.7 \%$ of non-psoriasis individuals. Validation of the psoriasis diagnosis yielded a high sensitivity and specificity, with little incremental value of limiting diagnoses to those diagnosed by a physician.

Conclusion The lifetime-prevalence of self-reported psoriasis was found to be $7.9 \%$, whereas the 1 -year prevalence (ie, currently active psoriasis) was $5.0 \%$. If used appropriately, questionnaire-based data may accurately identify patients with psoriasis.
\end{abstract}

\section{INTRODUCTION}

In the past decades there has been a dramatic increase in the number of studies that have investigated the epidemiology of common inflammatory skin diseases; either based on data routinely collected in clinics, observational data from administrative registries, or questionnaire-based data from general population or patient surveys. ${ }^{1-6}$ While these studies have predominantly focused on psoriasis, ${ }^{1-5}$ research in other skin diseases such as atopic dermatitis (AD), hidradenitis suppurativa and rosacea have also gained

\section{Strengths and limitations of this study}

Studies from Scandinavian countries have reported some of the highest psoriasis prevalence estimates in the world, but wide-ranging estimates could be explained by methodological differences and unvalidated outcome definitions. We validated the use of questionnaire data for psoriasis prevalence estimation, and demonstrated a high sensitivity and specificity of patient-reported psoriasis.

- To reduce the chance of participation bias, subjects were not informed about the content of the research project until they had agreed to participate.

- Questionnaire-based studies like the present one are subject to recall bias.

considerable momentum. ${ }^{7-13}$ Previous efforts to describe the disease prevalence, comorbidities, treatment and prognosis of psoriasis have overall led to increased awareness and understanding of the disease burden, however, studies have often yielded different and even conflicting results. ${ }^{14}$ For example, the prevalence of psoriasis has previously been assessed with great variation in Scandinavian countries, ranging from $2.2 \%$ to $11.4 \% .^{15-18}$ Several reasons may exist, including disease misclassification and recall-bias in retrospective questionnaire-based studies, ${ }^{19}$ differences in sample size, study design, as well as validity of the data and data sources. Moreover, large-scale epidemiological studies using routinely collected healthcare data often lack important information about disease-specific clinical characteristics and patient reported outcomes.

The present article describes the prevalence and clinical characteristics of patients with psoriasis, using baseline data from the Danish Skin Cohort, a prospective cohort of patients with psoriasis and AD in Denmark.

\section{MATERIALS AND METHODS}

All Danish residents have free, equal and universal healthcare access in Denmark. 
This, includes general practitioners, as well as specialists in private or hospital clinics. Patients initially consult their general practitioner, and from there they can be referred to a private or hospital dermatologist if the general practitioner finds it necessary, eg, to establish a firm diagnosis or provide certain treatments, for example, phototherapy. Referral does not necessitate severe disease.

\section{Danish skin cohort}

The Danish Skin Cohort is a prospective cohort established with the aim of studying the natural history and disease course of psoriasis and $\mathrm{AD}$ in Denmark. The Danish Skin Cohort is composed of three independent samples. Sample A comprised randomly sampled adults ( $\geq 18$ years) from the Danish general population. Sample B contained a group of adult patients with a clinical dermatologist verified diagnosis of plaque psoriasis. Sample C comprised a group of adult patients with a clinical dermatologist verified diagnosis of AD. Initially, the Civil Registration System ${ }^{20}$ was used to randomly select 10000 adults from the general population alive and resident in Denmark at time of cohort establishment (Sample A). The entire Danish population, aged 18 years or older, was eligible for selection. Second, the Danish National Patient Register ${ }^{21}$ was used to identify a total of 10000 adults with a clinician diagnosis of psoriasis (Sample B). The Danish National Patients Register contains information on all diagnoses given from public and private hospitals (including outpatient/ambulatory clinics), as well as diagnoses from a number of private practice dermatology clinics in Denmark. Patients with psoriasis were randomly selected among all patients that had at least one dermatologist diagnosis of psoriasis occurring in adulthood (ie, after their 18th birthday), regardless of whether or not this was their first-ever diagnosis. Third, a total of 10000 adults with a clinician diagnosis of $\mathrm{AD}$ were identified from the Danish National Patient Register (Sample C). To qualify, patients had to have at least one diagnosis of $A D$ verified by a dermatologist after their 18th birthday, regardless of whether or not this was their first-ever diagnosis. Importantly, to further reduce the chance of misclassification, patients with psoriasis were required to never had a recorded diagnosis of $\mathrm{AD}$, and vice versa for $\mathrm{AD}$ patients, regardless of the type diagnosing physician. To reduce the chance of participation bias, subjects were not informed about the content of the research project until they had agreed to participate. Consequently, the invited subjects were simply informed that the project was 'Research regarding the people in the Danish population' an were thus unaware that the content related to skin diseases. Upon accepting the invitation, and throughout the study, patients had the opportunity to withdraw from participation. A total of 3490 (adult general population), 4016 (adults with psoriasis) and 3834 (adults with AD), accepted the invitation to participate in the prospective cohort.

\section{Patient interviews}

Among the 30000 individuals that were invited to participate in the Danish Skin Cohort, those who accepted the invitation were systematically interviewed. In Denmark, all communications from the government and other official institutions are sent to a personal and secure digital mailbox, which citizens are obligated to check on a regular basis. Eligible subjects were sent an invitation to participate in the Danish Skin Cohort. In case of non-response, patients were sent a reminder after 1 week. Continued non-responders were contacted by phone or mail up to a total of five times. Subjects were interviewed in a structured manner by professional researchers over the phone, but had the option to answer the survey electronically if they preferred. Digital photographs were provided to all participants where applicable (eg, for assessment of Patient-Oriented SCORing Atopic Dermatitis. The survey was conducted between 15 May 2018 and 15 July 2018. Information on lifestyle and general health included height in $\mathrm{cm}$, weight in $\mathrm{kg}$, smoking history and quantity, current alcohol consumption and physical activity. Specific to skin diseases, patients were asked if they currently or at any point in time had psoriasis or $\mathrm{AD}$, and if they recalled whether a dermatologist, or any other type of physician had informed them about their diagnosis, respectively. Family history of psoriasis or AD was recorded, as was information about disease activity, including number of flares in the past 12 months, measurements of the currently affected body surface area (BSA). Use of patient-reported BSA has previously been validated and shown to accurately reflect physician-reported BSA scores. ${ }^{22}$ A flare was defined as one or more consecutive days with significant worsening of symptoms requiring escalation of treatment or seeking additional medical advice. ${ }^{23}$ Quantitative measures of touch avoidance, skin and joint pain, as well as pruritus, was obtained using a numeric rating scale (NRS). ${ }^{24-27}$ Information on Dermatology Life Quality Index (DLQI) was obtained regardless of whether they had ever had any skin disease.

\section{Statistical analysis}

Summary statistics were created and presented as frequencies with percentages for categorical variables and means with SDs for continuous variables. Furthermore, IQR were estimated for non-normally distributed continuous outcome variables. To validate the use of self-reported psoriasis, we calculated the sensitivity, specificity, positive and negative likelihood ratios of patient-reported psoriasis ('any psoriasis', 'physician diagnosed psoriasis' and 'dermatologist diagnosed psoriasis', respectively). As true-positive cases we used patients in the Danish Skin Cohort with dermatologist diagnosed psoriasis vulgaris (ie, the psoriasis sample), whereas patients with a dermatologist diagnosed $\mathrm{AD}$ (ie, the $\mathrm{AD}$ sample) were used as true-negative controls. By chart review of 100 patients with psoriasis and 100 patients with $\mathrm{AD}$, we have previously 


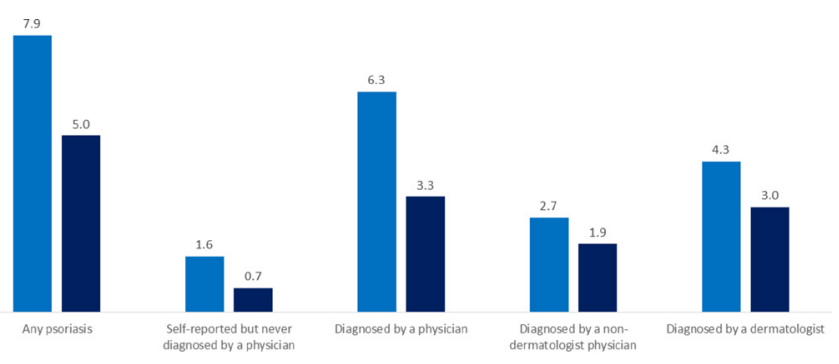

Figure 1 Prevalence of psoriasis in Denmark. Data were drawn from Sample A, that is, a general population sample of 3490 Danish adults.

validated these diagnoses with positive predictive values of $98 \%$ and $95 \%$, respectively. Importantly, during the validation of these diagnoses, no cases of psoriasis were reported among $\mathrm{AD}$ patients and vice versa. Analyses were performed using Stata software V.13.0.

\section{Patient and public involvement}

Patients and or public were not involved in the design or conduct of the current study.

\section{Data sharing}

There is no plan to share raw data from this study.

\section{RESULTS}

In the following, only findings from the baseline assessment of the general population sample (Sample A) are reported. Data on the entire invited population and non-responders are provided in online supplementary table 1 . Out of a total of 3490 general population participants, 41 individuals (1.2\%) were excluded from the study (values treated as missing), since they did not wish to participate after being informed that the study was focused on skin diseases. Overall, the prevalence of self-reported psoriasis $(\mathrm{n}=275)$ in the general population was $7.9 \%$ (95\% CI $7.0 \%$ to $8.8 \%$ ), with an age and sex distribution comparable to that of the general population participants without self-reported psoriasis. A total of 221 $(80.4 \%)$ of psoriasis patients reported that their disease had been diagnosed by a physician (predominantly

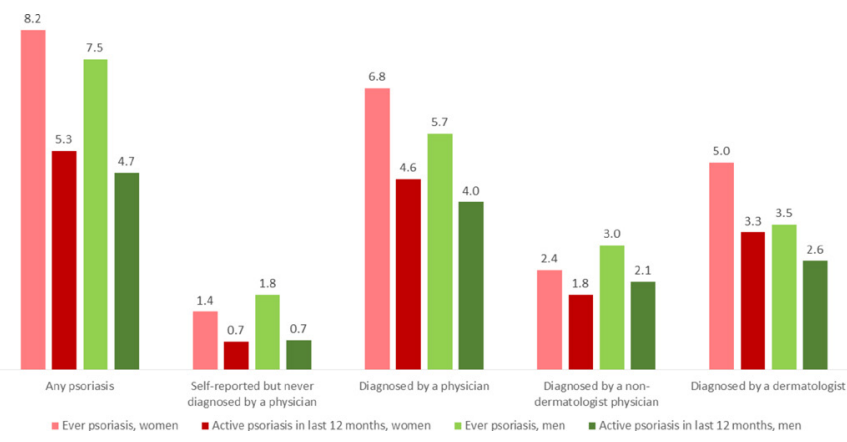

Figure 2 Sex-specific prevalence of psoriasis in Denmark. Data were drawn from Sample A, that is, a general population sample of 3490 Danish adults. dermatologists, [151 of 221]), whereas $54(19.6 \%)$ of the population reported having undiagnosed psoriasis, yielding a prevalence of $1.6 \%$ for undiagnosed psoriasis (figure 1). When limited to active psoriasis within the last 12 months, the prevalence of psoriasis was $5.0 \%(n=176)$. Stratified by sex, the prevalence of psoriasis was $8.2 \%$ and $7.5 \%$ among women and men, respectively. Active psoriasis within the last 12 months was reported by $5.3 \%$ of women, and $4.7 \%$ of men (figure 2 ).

\section{Physician diagnosed psoriasis among general population adults}

Among the total 221 individuals reporting physician-diagnosed psoriasis, there was a strong female predominance $(59.7 \%)$ as shown in table 1 . This gender disproportionality was driven by dermatologist-diagnosed psoriasis (63.6\% women vs $36.4 \%$ men) whereas the sex-distribution was equal among patients diagnosed by non-dermatologists ( $51.4 \%$ women vs $48.6 \%$ men). The majority of patients had mild disease $(63.8 \%)$ with a current BSA less than 3. Roughly one in every 10 patients $(11.8 \%)$ had severe psoriasis, ie, a BSA of 10 or greater. Of note, $46.6 \%$ reported having at least one family member with psoriasis, with $12.2 \%$ of patients having more than one family member with psoriasis. The mean (SD) DLQI was 2.3 (3.6), and the NRS score $(0=$ no pain, $10=$ worst pain imaginable) for joint pain was 3.3 (2.8). One-third of patients $(37.6 \%)$ reported stable disease, ie, no disease flares in the past 12 months.

\section{Self-reported psoriasis among general population adults}

Among patients responding that they had psoriasis that had never been diagnosed by a physician, there was an equal sex distribution. Interestingly, a family history of psoriasis was lower among patients with self-reported psoriasis than among dermatologist-verified psoriasis patients, although that a positive family history was still twice as frequent as in non-psoriasis individuals. In total $44.4 \%(n=24)$ of these patients reported that there had been visible psoriasis lesions within the last 12 months, and their median DLQI was 3 (IQR 1-4) versus 0 (IQR $0-1$ ) among patients with self-reported psoriasis that did not have visible lesions within the last 12 months. Joint pain was comparable (mean NRS 3.2 vs 3.3) among patients with self-reported and physician diagnosed psoriasis, respectively. Generally, patients with self-reported psoriasis had more stable disease, ie, less disease flares, an a numerically higher occurrence of mild psoriasis, compared with physician-reported psoriasis, respectively. A list of questions obtained during the survey is shown in online Supplementary table 2 .

\section{Validating the use of a questionnaire-based approach for identification of psoriasis}

To examine the validity of questions aiming at identifying psoriasis, the dermatologist diagnosed psoriasis sample of 4016 patients (Sample B) and the dermatologist diagnosed AD sample of $3834 \mathrm{AD}$ patients (Sample C) were 
Table 1 Characteristics of people with psoriasis from an adult general population sample

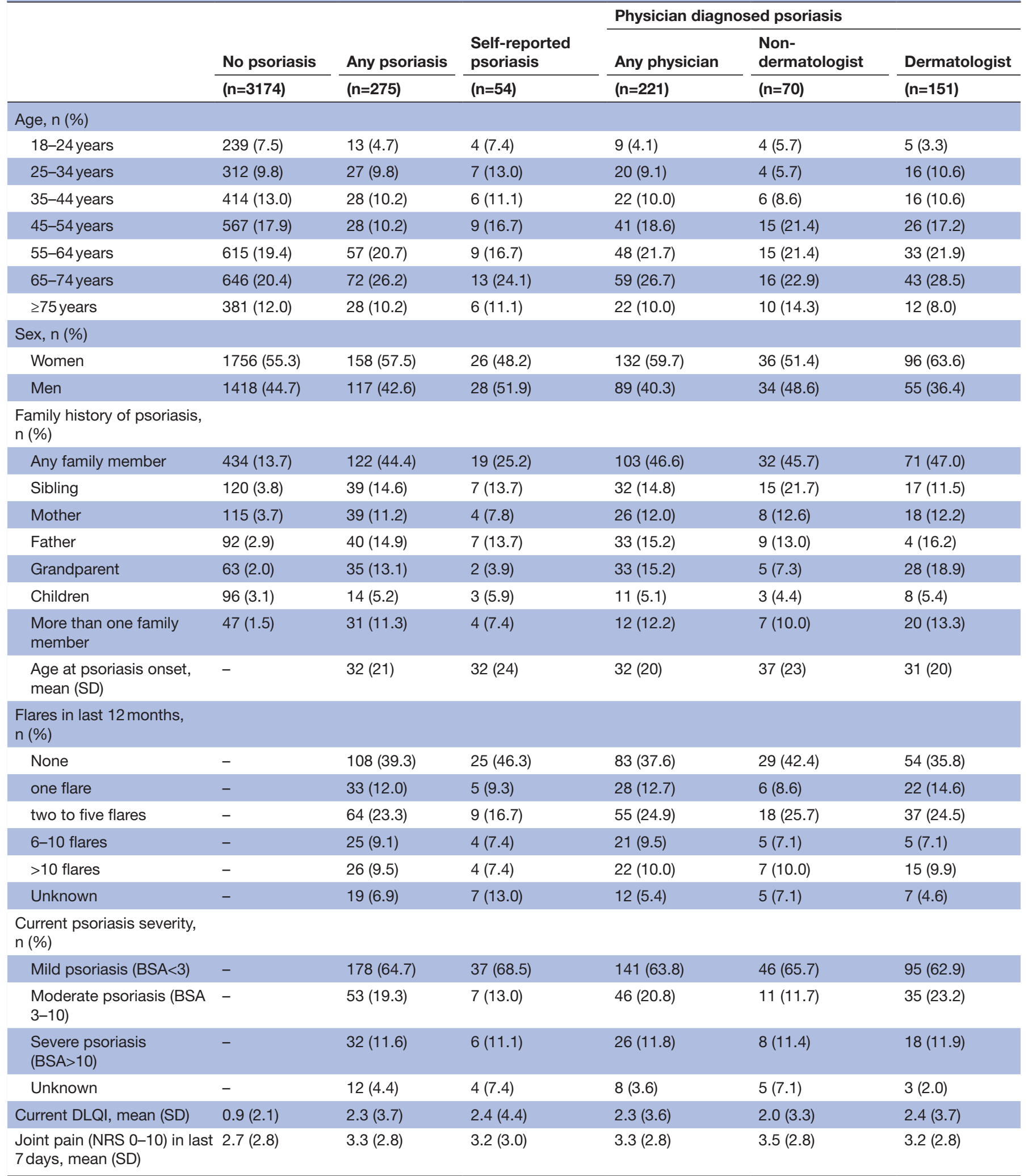

BSA, body surface area; DLQI, Dermatology Life Quality Index; NRS, numerical rating scale.

used. The sensitivity of patient-reported psoriasis ('Have you ever had psoriasis?') was $84.4 \%$ (95\% CI $83.2 \%$ to $85.5 \%$ ) and the specificity was $88.2 \%$ (95\% CI $87.1 \%$ to $89.2 \%)$. Asking specifically about physician-diagnosed psoriasis ('Has a doctor ever told you that you have/had psoriasis?') yielded a comparable sensitivity and specificity (table 2). Asking patients 'Has a dermatologist ever told you that you have/had psoriasis?' yielded the a sensitivity 
Table 2 Validation of the use of questionnaire-based surveys for identification of psoriasis

\begin{tabular}{|c|c|c|c|c|c|c|c|c|}
\hline & $\begin{array}{l}\text { Sensitivity } \\
\text { (\%) }\end{array}$ & $95 \% \mathrm{Cl}$ & $\begin{array}{l}\text { Specificity } \\
\text { (\%) }\end{array}$ & $95 \% \mathrm{Cl}$ & LR+ & $95 \% \mathrm{Cl}$ & LR- & $95 \% \mathrm{Cl}$ \\
\hline $\begin{array}{l}\text { Patient-reported } \\
\text { psoriasis (regardless } \\
\text { of whether or not this } \\
\text { was diagnosed by a } \\
\text { physician) }\end{array}$ & 84.4 & 83.2 to 85.5 & 88.2 & 87.1 to 89.2 & 7.14 & 6.54 to 7.80 & 0.18 & 0.16 to 0.19 \\
\hline $\begin{array}{l}\text { Patient-reported } \\
\text { physician diagnosed } \\
\text { psoriasis }\end{array}$ & 83.3 & 82.2 to 84.5 & 91.8 & 90.8 to 92.6 & 10.11 & 9.08 to 11.26 & 0.18 & 0.17 to 0.20 \\
\hline $\begin{array}{l}\text { Patient-reported } \\
\text { dermatologist } \\
\text { diagnosed psoriasis }\end{array}$ & 78.8 & 77.6 to 80.10 & 93.6 & 92.8 to 94.4 & 12.31 & 10.90 to 13.91 & 0.23 & 0.21 to 0.24 \\
\hline
\end{tabular}

True positive cases were drawn from Sample B. True negative cases were drawn from Sample C.

Sensitivity $=$ true positive / (true positive + false negative).

Specificity $=$ true negative $/$ (false positive + true negative).

$\mathrm{LR}+=$ sensitivity / ( 1 - specificity).

LR- = $(1-$ sensitivity $) /$ specificity.

$\mathrm{LR}+$, positive likelihood ratio; LR-, negative likelihood ratio.

of 78.8 (95\% CI $77.6 \%$ to $80.1 \%$ ), with a specificity of $93.6 \%$ (95\% CI $92.8 \%$ to $94.4 \%$ ).

\section{DISCUSSION}

In this population-based study of adult Danes, the lifetime prevalence of self-reported psoriasis was $7.9 \%$, whereas the 1 year period prevalence of was $5.0 \%$. The majority of patients reported one or more disease flares within the last 12 months, but the majority of patients had mild disease. Noticeable differences were seen based on diagnostic methodology (self-diagnosed, physician diagnosed or dermatologist diagnosed), yet use of a questionnaire-based approach for identification of patients with psoriasis generally yielded high sensitivity, and specificity, with little added value of limiting the question to whether psoriasis had been diagnosed by a physician or dermatologist.

In our study, there was a strong female predominance among patients reporting dermatologist-diagnosed psoriasis, whereas the male-to-female ratio was more balanced (and comparable to the general population) in the overall group of patients diagnosed solely by non-dermatologists (eg, general practitioners). Although speculative, this could be explained by differences in healthcare seeking behaviour, whereby women may be more prone to consult a physician about skin problems and furthermore request a referral to a dermatologist for treatment of their psoriasis. In agreement with our findings, a previous surveybased study examined the prevalence of psoriasis among 3471 residents in the Copenhagen between 2006-2008 using the question 'Have you ever been told by a physician that you had psoriasis?', and found a prevalence of $7.1 \%{ }^{16}$ Interestingly, while the study did not distinguish between the type of diagnosing physician, $57 \%$ of the affirmative responders were woman, versus $55 \%$ of sampled individuals without psoriasis. In contrast, a recent
European questionnaire-based study showed that overall, the same proportions of men and women with psoriasis were treated by general physicians and dermatologists. ${ }^{28}$ However, stratification by country showed considerable differences in access to dermatologists.

Our findings in the validation study support that survey data may adequately identify adult patients with psoriasis, although that prevalence estimates may tend to slightly underestimate the true prevalence as evident by the sensitivity in our validation study. This is in stark contrast to $\mathrm{AD}$, where survey-based data are generally considered inapplicable in adult population due to very high risk of misclassification. ${ }^{19}$ Previously, registry-based studies have suggested a somewhat lower prevalence of psoriasis in Denmark and other European countries such as the UK. ${ }^{1529}$ While registry based studies using routinely collected administrative data may hold information on large numbers of patients, such studies most often require that patients consult their physicians in order to be accurately captured. Accordingly, such studies may tend to underestimate the true prevalence, in particular since family knowledge about psoriasis and its treatment may prevent for example, men from seeking medical help. ${ }^{17}$ In general, questionnaire-based studies may suffer from non-response, sampling bias and misclassification leading to a false-high prevalence.

Certain strength and limitations apply to the methodology, robustness and interpretation of these data. In our study, the majority of patients reporting a history of psoriasis were diagnosed by a physician, whereas a smaller proportion reported a positive history of self-diagnosed psoriasis. Interestingly, a positive family history was more frequent in these patients than in non-psoriasis individuals, but remained conspicuously more uncommon when compared with patients with physician-diagnosed psoriasis. Indeed, this could suggest that patients with 
other skin diseases may incorrectly believe that they have psoriasis. Notably, unlike for example, the USA, where advertisements with pictures of psoriasis are frequently displayed on television and in newspapers, pharmaceutical advertisements towards non-healthcare professionals are not allowed in Denmark. Thus, Danish residents may be less likely to recognize psoriasis lesions, which could potentially lead to disease misclassification if they never consult a physician about their skin condition. Our specificity estimates were calculated using a cohort of patients with $\mathrm{AD}$ as true-negative controls, which may slightly limit the generalisability to the general population. The Danish Skin Cohort originally had 30000 people invited to participate, and approximately $40 \%$ of people agreed to be enrolled. This is in line with response rates from other studies, in comparable populations. ${ }^{30}$ Importantly, our cohort was comparable to the general population in terms of baseline demographics suggesting that results may be generalisable on a national level. Furthermore, since only $1.2 \%$ of patients declined to participate, we feel fairly confident that our study was not significantly affected by participation bias (ie, patients with skin disease being more willingly to participate). Nevertheless, some degree of selection bias may have occurred, as the age and gender distribution of responders compared with invitees were marginally skewed (online supplementary table 1). Furthermore, recall bias could have affected the results, since we only surveyed adults, some may have consulted a physician in childhood due to psoriasis or not even remember it. Furthermore, patients may have received treatment for their psoriasis by their physician without the physician actually telling them that they in fact had psoriasis.

\section{CONCLUSION}

We found a life-time self-reported psoriasis prevalence of $7.9 \%$ (95\% CI $7.0 \%$ to $8.8 \%$ ) in Denmark, whereas the 1 year period prevalence was $5.0 \%$. Physician-diagnosed psoriasis had a lifetime prevalence of $6.3 \%$, and a 1 year period prevalence of $3.3 \%$. The majority of patients had mild disease, although approximately two-thirds of patients had at least one psoriasis flare in the last 12 months. Our data showed that questionnaire-based assessment of psoriasis prevalence is a valid approach with a high sensitivity and specificity.

Contributors AE had full access to all of the data in the study and take responsibility for the integrity of the data and the accuracy of the data analysis. Study concept and design: AE, YMFA, JPT. Acquisition, analysis and interpretation of data: AE, YMFA, JPT. Drafting of the manuscript: AE. Critical revision of the manuscript for important intellectual content: AE, YMFA, JPT. Statistical analysis: AE. Obtained funding: None. Administrative, technical or material support: AE. Study supervision: AE. Substantial contributions to the conception or design of the work and/or to acquisition, analysis or interpretation of data for the work: AE, YMFA, JPT. Drafting the work or revising it critically for important intellectual content: $A E$, YMFA, JPT. Final approval of the version to be published: AE, YMFA, JPT. Agreement to be accountable for all aspects of the work in ensuring that questions related to the accuracy or integrity of any part of the work are appropriately investigated and resolved: AE, YMFA, JPT.
Funding Supported by funding from the Danish Psoriasis Foundation (Psoriasisforeningen).

Disclaimer Funding agency/sponsor was not involved in the design and conduct of the study; collection, management, analysis and interpretation of data; preparation, review or approval of the manuscript or the decision to submit the manuscript for publication.

Competing interests None declared.

Patient consent for publication Not required.

Ethics approval All relevant study approvals were obtained (ref. 2012-58-0004, j.no. VD-2018-286, I-Suite no.: 6528). Review of an ethics committee is not required in Denmark for studies not involving human tissue.

Provenance and peer review Not commissioned; externally peer reviewed. Data sharing statement № additional data are available.

Open access This is an open access article distributed in accordance with the Creative Commons Attribution Non Commercial (CC BY-NC 4.0) license, which permits others to distribute, remix, adapt, build upon this work non-commercially, and license their derivative works on different terms, provided the original work is properly cited, appropriate credit is given, any changes made indicated, and the use is non-commercial. See: http://creativecommons.org/licenses/by-nc/4.0/.

\section{REFERENCES}

1. Gelfand JM, Neimann AL, Shin DB, et al. Risk of myocardial infarction in patients with psoriasis. JAMA 2006;296:1735-41.

2. Mallbris L, Akre O, Granath F, et al. Increased risk for cardiovascular mortality in psoriasis inpatients but not in outpatients. Eur $J$ Epidemiol 2004;19:225-30.

3. Wu JJ, Choi YM, Bebchuk JD. Risk of myocardial infarction in psoriasis patients: a retrospective cohort study. J Dermatolog Treat 2015;26:230-4.

4. Ahlehoff O, Gislason $\mathrm{GH}$, Charlot M, et al. Psoriasis is associated with clinically significant cardiovascular risk: a Danish nationwide cohort study. J Intern Med 2011;270:147-57.

5. Egeberg A, Thyssen JP, Jensen P, et al. Risk of myocardial infarction in patients with psoriasis and psoriatic arthritis: a nationwide cohort study. Acta Derm Venereol 2017;97:819-24.

6. Silverberg Jl. Association between adult atopic dermatitis, cardiovascular disease, and increased heart attacks in three population-based studies. Allergy 2015;70:1300-8.

7. Andersen YM, Egeberg A, Gislason GH, et al. Risk of myocardial infarction, ischemic stroke, and cardiovascular death in patients with atopic dermatitis. J Allergy Clin Immunol 2016;138:310-2.

8. Standl M, Tesch F, Baurecht $\mathrm{H}$, et al. Association of atopic dermatitis with cardiovascular risk factors and diseases. J Invest Dermatol 2017; 137:1074-81.

9. Marshall VD, Moustafa F, Hawkins SD, et al. Cardiovascular disease outcomes associated with three major inflammatory dermatologic diseases: a propensity-matched case control study. Dermatol Ther 2016;6:649-58.

10. Egeberg A, Hansen PR, Gislason GH, et al. Exploring the association between rosacea and parkinson disease: a Danish nationwide cohort study. JAMA Neurol 2016;73:529-34.

11. Egeberg A, Gislason GH, Hansen PR. Risk of Major Adverse Cardiovascular Events and All-Cause Mortality in Patients With Hidradenitis Suppurativa. JAMA Dermatol 2016;152:429-34.

12. Egeberg A, Jemec GBE, Kimball AB, et al. Prevalence and risk of inflammatory bowel disease in patients with hidradenitis suppurativa. J Invest Dermatol 2017;137:1060-4.

13. Egeberg A, Ashina M, Gaist D, et al. Prevalence and risk of migraine in patients with rosacea: a population-based cohort study. J Am Acad Dermatol 2017;76:454-8.

14. Thyssen JP, Halling-Overgaard AS, Andersen YMF, et al. The association with cardiovascular disease and type 2 diabetes in adults with atopic dermatitis: a systematic review and meta-analysis. $\mathrm{Br} \mathrm{J}$ Dermatol 2018;178:1272-9.

15. Egeberg A, Skov L, Gislason GH, et al. Incidence and prevalence of psoriasis in Denmark. Acta Derm Venereol 2017;97:808-12.

16. Jensen $P$, Thyssen JP, Zachariae $C$, et al. Cardiovascular risk factors in subjects with psoriasis: a cross-sectional general population study. Int J Dermatol 2013;52:681-3.

17. Gjersvik P. Psoriasis is More Prevalent than Indicated by Egeberg et al.'s Danish Study: a comment. Acta Derm Venereol 2018;98:167-8.

18. Danielsen $\mathrm{K}$, Olsen AO, Wilsgaard T, et al. Is the prevalence of psoriasis increasing? A 30-year follow-up of a population-based cohort. Br J Dermatol 2013;168:1303-10. 
19. Andersen YMF, Egeberg A, Hamann CR, et al. Poor agreement in questionnaire-based diagnostic criteria for adult atopic dermatitis is a challenge when examining cardiovascular comorbidity. Allergy 2018;73:923-31.

20. Schmidt M, Pedersen L, Sørensen HT. The Danish Civil Registration system AS a tool in epidemiology. Eur J Epidemiol 2014;29:541-9.

21. Schmidt M, Schmidt SA, Sandegaard JL, et al. The Danish National patient registry: a review of content, data quality, and research potential. Clin Epidemiol 2015;7:449-90.

22. Housman TS, Patel MJ, Camacho F, et al. Use of the SelfAdministered Eczema Area and Severity Index by parent caregivers: results of a validation study. $\mathrm{Br} J$ Dermatol 2002;147:1192-8.

23. Langan SM, Thomas KS, Williams HC. What is meant by a "flare" in atopic dermatitis? A systematic review and proposal. Arch Dermatol 2006;142:1190-6.

24. Ferreira-Valente MA, Pais-Ribeiro JL, Jensen MP. Validity of four pain intensity rating scales. Pain 2011;152:2399-404.
25. Kimball AB, Naegeli AN, Edson-Heredia E, et al. Psychometric properties of the itch numeric rating scale in patients with moderateto-severe plaque psoriasis. Br J Dermatol 2016;175:157-62.

26. Humphrey L, Symonds T, Gable J, et al. Validity and interpretation of a skin pain numeric rating scale among adults and adolescents with atopic dermatitis. Value in Health 2017;20:A806.

27. Humphrey L, Symonds T, Gable J, et al. Mixed methods evaluation of an itch numeric rating scale among adult and adolescent patients with atopic dermatitis. Value in Health 2017;20:A806.

28. Svensson A, Ofenloch RF, Bruze M, et al. Prevalence of skin disease in a population-based sample of adults from five European countries. Br J Dermatol 2018;178:1111-8.

29. Springate DA, Parisi R, Kontopantelis E, et al. Incidence, prevalence and mortality of patients with psoriasis: a U.K. population-based cohort study. Br J Dermatol 2017;176:650-8.

30. Modalsli EH, Snekvik I, Åsvold BO, et al. Validity of self-reported psoriasis in a general population: the HUNT Study, Norway. J Invest Dermatol 2016;136:323-5. 